\title{
Medidas de bioseguridad y miedo a la COVID-19 asociado a calidad de vida en el trabajo en personal asistencial de salud de un hospital
}

\author{
Biosecurity measures and fear of COVID-19 associated with quality of life \\ at work in healthcare personnel in a hospital \\ Medidas de biossegurança e medo do COVID-19 associado à qualidade de vida \\ no trabalho em pessoal de saúde hospitalar
}

Antony Alejandro Llerena Torrejón

Obed Eleazar López Condori'

\section{Resumen}

Objetivo: Determinar la relación entre medidas de bioseguridad y miedo a la COVID-19 con la calidad de vida en el trabajo en personal asistencial de salud. Material y métodos: Estudio analítico transversal, se incluyó a 245 trabajadores asistenciales del Hospital Huaycán en Lima, Perú, quienes laboraron durante la pandemia de COVID-19. Se aplicó una encuesta online, durante el año 2021, compuesta por tres cuestionarios: medidas de bioseguridad, Fear of COVID-19 Scale para evaluar miedo y otro para medir calidad de vida en el trabajo. Se utilizó prueba Chi-cuadrado, T de Student, Odds Ratio (OR) con intervalo de confianza (IC) al $95 \%$, se consideró significativo cuando $p<0,05$. Resultados: Los trabajadores presentaron media de edad de 38,6 \pm 8,5 años, donde el 68,2 \% son mujeres. El 61,6 \% sí cumple medidas de bioseguridad en prevención de la COVID-19, el 81,6\% presentan mala calidad de vida en el trabajo, el 43,7\% tienen mucho miedo a la COVID-19 y el 48,2\% tiene miedo a perder la vida por COVID-19. El personal que tuvo miedo cuando ve noticias o historias sobre la COVID-19 tuvo 6 veces más riesgo de presentar mala calidad de vida en el trabajo (OR: 6,76; IC 95 \%: 1,51-30,12), quienes no pueden dormir por estar preocupados por la COVID-19 tuvieron 5 veces más riesgo de presentar mala calidad de vida en el trabajo (OR: 5,51; IC 95 \%: 1,10- 27,49). Conclusiones: El personal de salud usa regularmente medidas de bioseguridad, la mitad tienen miedo a la COVID-19; la mayoría tienen mala calidad de vida, la que se asocia al miedo por ver noticias o historias sobre la COVID-19 y no pueden dormir por estar preocupados por la COVID-19.

Palabras clave: medidas de bioseguridad, miedo a la COVID-19, calidad de vida en el trabajo, trabajadores de salud

\section{Abstract}

Objective: To determine the relationship between biosecurity measures and fear of COVID-19 with quality of life at work in healthcare personnel. Material and methods: Crosssectional analytical study, 245 healthcare workers from the Huaycán Hospital in Lima Peru, who worked during the COVID-19 pandemic, were included. An online survey was applied, during the year 2021, consisting of three questionnaires: biosafety measures, the Fear of COVID-19Scale to assess fear and another to measure quality of life at work. Chi-square test, Student's t test, Odds Ratio (OR) with $95 \%$ confidence interval (Cl) was used, it was considered significant when $\mathrm{p}<0.05$. Results: The workers had an average age of 38.6 \pm 8.5 years, where $68.2 \%$ were women. $61.6 \%$ do comply with biosecurity measures in prevention of COVID-19, $81.6 \%$ have poor quality of life at work, $43.7 \%$ are very afraid of COVID-19 and 48.2 $\%$ are afraid of losing their lives due to COVID-19. Staff who were afraid when watching news or stories about COVID-19 were 6 times more at risk for poor quality of life at work (OR: 6.76; 95 \% Cl: 
1.51-30.12), who cannot sleep because they are worried about COVID-19 had 5 times more risk of poor quality of life at work (OR: 5.51; $95 \%$ Cl: 1.1027.49). Conclusion: Health workers regularly use biosecurity measures, half are afraid of COVID-19; most have poor quality of life, which is associated with fear of seeing news or stories about COVID19 and cannot sleep because they are worried about COVID-19.

Keywords: biosecurity measures, fear of COVID19, quality of life at work, health workers

\section{Resumo}

Objetivo: Determinar a relação entre as medidas de biossegurança e o medo da COVID-19 com a qualidade de vida no trabalho em pessoal de saúde. Material e métodos: Estudo analítico transversal, foram incluídos 245 trabalhadores assistenciais do Hospital Huaycán em Lima, Peru, que trabalharam durante a pandemia de COVID19. No ano de 2021, foi realizado um inquérito online, composto por três questionários: medidas de biossegurança, Fear of COVID-19 Scale para avaliar o medo e outro para medir a qualidade de vida no trabalho. Foi utilizado o teste Qui-quadrado, T de Student, Odds Ratio (OR) com intervalo de confiança (IC) a $95 \%$, considerado significativo quando $p<0,05$.

Resultados: Os trabalhadores apresentaram média de idade de 38,6 \pm 8,5 anos, onde 68,2 \% são mulheres. 61,6 \% cumprem as medidas de biossegurança para a prevenção da COVID-19, $81,6 \%$ têm uma má qualidade de vida no trabalho, 43,7 \% têm muito medo da COVID-19 e 48,2 \% receiam perder a vida devido à COVID-19. O pessoal que teve medo quando viu notícias ou histórias sobre a COVID-19 teve 6 vezes mais risco de apresentar má qualidade de vida no trabalho (OR: 6,76; IC 95 \%: 1,51-30,12), que não podem dormir por estarem preocupados com a COVID19 tiveram 5 vezes mais risco de apresentar má qualidade de vida no trabalho (OR: 5,51; IC 95 \%: 1,10-27,49). Conclusão: $O$ pessoal de saúde usa regularmente medidas de biossegurança, metade tem medo da COVID-19; a maioria tem má qualidade de vida, a que se associa ao medo de ver notícias ou histórias sobre a COVID-19 e não podem dormir por estarem preocupados com a COVID-19.
Palavras-chave: medidas de biossegurança, medo da COVID-19, qualidade de vida no trabalho, trabalhadores da saúde

\section{Introducción}

Al 15 de abril de 2021, la Organización Mundial de la Salud (OMS)' reporta 138 millones de casos confirmados y 2,97 millones de muertes por coronavirus (COVID- 19) en el mundo. De igual manera la Organización Panamericana de la Salud (OPS) ${ }^{2}$ informa que en América Latina y el Caribe existen 26571000 casos, con un total de 841000 muertos. El Perú ocupa el quinto lugar dentro de los países con mayor incidencia de esta enfermedad, con un total de aproximadamente 1,66 millones de casos y 55489 muertos hasta la fecha.

En torno a los profesionales de la salud, la OPS/OMS estiman que 570000 profesionales de la región se han enfermado y más de 2500 han sucumbido ante el virus. ${ }^{4}$ Asimismo, en el Perú, se ha informado que un total de 5400 profesionales se han contagiado de COVID-19, y que alrededor de 300 han fallecido. ${ }^{5}$ Estas cifras exigen orientación sobre procedimientos de bioseguridad adecuados para proteger al personal sanitario, especialmente cuando las infecciones y las cuarentenas agravan la escasez de personal y de equipo de protección personal (EPP) e imponen una carga adicional a los servicios sanitarios ya colapsados. ${ }^{6}$ Múltiples estudios confirmaron que el acceso a un EPP y la implementación adecuada de protocolos de bioseguridad favorecen el sentimiento de seguridad de los profesionales de salud. Con suficiente EPP, los trabajadores se sienten más protegidos de los contagios, lo que puede disminuir su ansiedad y el miedo a infectar a sus seres queridos.

Por otro lado, el profesional de salud es el encargado de identificar a las personas contagiadas con la enfermedad, dar respuesta a sus necesidades de tratamiento, realizar los severos y difíciles procesos de tratamiento en los pacientes hospitalizados, afrontar el colapso psicológico que genera cada paciente fallecido y también afrontar el riesgo de desarrollar la 
enfermedad en cualquier momento. Cada situación mencionada es una condición de vida difícil en sí misma, y se espera que estas condiciones generen consecuencias psicosociales a corto y largo plazo para los profesionales de la salud.

Aunque no hay datos epidemiológicos definitivos sobre los efectos psicológicos del COVID-19 en las personas y su efecto en la salud pública, los resultados de los estudios limitados muestran que el miedo a contraer COVID-19 conduce a intensas consecuencias emocionales y conductuales como el aburrimiento, soledad, ansiedad, problemas para dormir e ira. ${ }^{10,11}$ Teniendo en cuenta que el profesional sanitario también puede ser susceptible a diversas afecciones psicopatológicas, se puede pensar que la situación de riesgo potencial aumentará aún más el riesgo de depresión, trastornos de ansiedad, trastorno de estrés postraumático (TEPT), trastornos paranoicos y psicóticos, e incluso el suicidio. ${ }^{12}$ Las experiencias que quedan de brotes anteriores, como el ébola, también apoyan esta opinión. 13,14 Incluso si los períodos pandémicos han terminado, se espera que ocurran efectos psicosociales secundarios en los profesionales de la salud que experimentan un trauma de cerca, y pueden afectar su calidad de vida. Por tanto, es de esperar que el miedo que desarrollen los profesionales sanitarios en este proceso repercuta negativamente en sus habilidades de ajuste psicológico al desencadenar diversos síntomas psicopatológicos.

Dada la novedad de la crisis por COVID-19, es necesario realizar investigaciones que permitan recopilar datos sobre la calidad de vida de los trabajadores de salud y los factores que puedan estar influyendo en ella, como el miedo y las medidas de bioseguridad, para proponer estrategias de intervención a futuro. ${ }^{16}$

\section{Material y métodos}

El diseño del estudio fue analítico, transversal y se realizó en profesionales de la salud del Hospital II1 Huaycán que se encuentra ubicado en el distrito de Ate y pertenece a la Red Metropolitana
Lima-Este. Se incluyó a 245 profesionales de la salud (médicos, enfermeros, obstetras, tecnólogos médicos) y técnicos, que se encuentran laborando en la institución desde que inició la pandemia, que completaron los cuestionarios correctamente y que aceptaron participar en la investigación, previo consentimiento informado. Se excluyeron a personas que no hayan laborado en la institución durante la pandemia.

La técnica empleada fue la encuesta online y el instrumento el cuestionario. Para medir la variable medidas de bioseguridad, se elaboró un cuestionario basado en el documento técnico "Plan nacional de reforzamiento de los servicios de salud y contención del COVID-19"17 y el "Plan para la vigilancia, prevención y control de COVID19 en el trabajo" ${ }^{\prime 18}$ basados en la Resolución Ministerial 95-2020-MINSA. El instrumento contó con 18 ítems con alternativas de respuesta dicotómicas de Sí y No. La escala final de puntuación clasifica las medidas de bioseguridad en cumple (13-18 puntos) y no cumple (1-12 puntos).

Para la validez del instrumento se realizó el juicio de expertos, que incluyó a un médico infectólogo y a la jefa del área de epidemiología del hospital de estudio y al jefe médico del área UCI-COVID de un Hospital de la Seguridad Social. Se obtuvo un valor de $V$ de Aiken de 0,81, que revela muy alta validez. Asimismo, para la confiabilidad se realizó un piloto con 20 profesionales de la salud que no fueron considerados dentro de la muestra de estudio. Se halló un coeficiente de KR-20 para respuestas dicotómicas de 0,86, que demostró su alta confiabilidad.

Por otro lado, para la variable miedo al COVID-19, se empleó el cuestionario "Fear of COVID-19 Scale" creado por Ahorsu et al. ${ }^{19}$ en Irán y validado en el Perú por Huarcaya et al. ${ }^{20}$ en el 2020 con una confiabilidad por alfa de Cronbach de 0,87.

El instrumento cuenta con 7 ítems divididos en 2 dimensiones: reacciones emocionales al miedo (1,2,4,5 ítems) y expresiones somáticas al miedo (3,6,7 ítems). Las alternativas de respuesta son 
tipo Likert y van desde el 1 (totalmente en desacuerdo) hasta el 5 (totalmente de acuerdo). De igual manera, la escala de puntuación final clasifica el miedo a la COVID-19 en: sí tiene y no tiene.

Por último, para la variable calidad de vida en el trabajo, se utilizó el cuestionario creado por Moreno, Cuevas y González en Puerto Rico en el $2009^{21}$ con una confiabilidad por alfa de Cronbach de 0,7. El instrumento cuenta con 46 ítems divididos en 8 dimensiones: condiciones del trabajo (1-21 ítems), exigencias trabajo-vida (22-28 ítems), clima hacia la seguridad (29-32 ítems), discriminación (33-35 ítems), ergonomía (36-39 ítems), salarios y beneficios (40-42 ítems), hostigamiento (43, 44 ítems) y flexibilidad $(45,46$ ítems). Las opciones de respuesta son de tipo Likert y van desde 1 (nunca) hasta 4 (siempre). Asimismo, la escala de puntuación final clasifica la calidad de vida en el trabajo en buena (124-167 puntos) y mala (80-123 puntos).

Para el análisis, se utilizó estadística descriptiva y analítica, para las variables categóricas se presenta frecuencias absolutas y relativas y para variables cuantitativas se presenta medias con su desviación estándar. Para la comparación de variables categóricas se utilizó la prueba Chicuadrado y para la comparación de variables cuantitativas la prueba $\mathrm{T}$ de Student, considerando asociación cuando el valor de $p$ fue menor de 0,05. Además, se utilizó Odds Ratio (OR) con intervalo de confianza (IC) al $95 \%$, considerando riesgo cuando el IC fue mayor de 1.

\section{Resultados}

De un total de 245 profesionales de salud encuestados, el 68,2\% fueron mujeres y el 31,8\% varones, con una edad promedio de 38,6 $\pm 8,5$ años. Asimismo, el 62 \% señaló estar casado o ser conviviente y el $38 \%$ se encontraba soltero o divorciado. En torno a las características laborales, el 45,7 \% fueron licenciados (enfermeros, obstetras, tecnólogos médicos), el $59,2 \%$ contaban con un contrato CAS o de tercerización y la mayoría llevaba 7,1 \pm 5,9 años laborando en la institución. Además, el 62,4\% laboraba en unidades no críticas y $64,9 \%$ manifestó contar con un segundo trabajo (Tabla 1).

En el análisis descriptivo de las variables de estudio, se observa que el 61,6\% de los profesionales de salud sí cumple con las medidas de bioseguridad para la prevención del COVID-19, mientras que el 38,4\% no las cumple. Con respecto a la calidad de vida en el trabajo, el 81,6\% de los encuestados la calificó como mala y el 18,4\% como buena, la misma tendencia se observa al análisis de sus dimensiones (Tabla 2).

En relación al miedo por COVID-19, el análisis se hace por pregunta, y se encontró que el 43,7\% tiene mucho miedo a la COVID-19 y el $48,2 \%$ tiene miedo a perder la vida por el COVID-19. En el resto de preguntas, la mayoría señaló no tener miedo (Tabla 2).

En el análisis bivariado, se encontró que los encuestados con una buena calidad de vida en el trabajo tuvieron una edad promedio de $36,1 \pm$ 9,04 años; mientras que, el grupo con una mala calidad de vida tuvo una edad de 39,2 \pm 8,3 años $(p=0,025)$. Seguidamente, se hallaron diferencias entre la calidad de vida en el trabajo y los ítems "Mis manos se ponen húmedas cuando pienso en el COVID-19" ( $p=0,008$ ), "Cuando veo noticias e historias sobre el COVID19 en redes sociales me pongo nervioso" ( $p<$ $0,01)$ y "No puedo dormir porque estoy preocupado de contagiarme del COVID-19" $(p=$ 0,001) de la variable miedo al COVID-19 (Tabla 3).

En el análisis multivariado, se encontró que los profesionales de la salud que tienen miedo cuando ven noticias o historias sobre la COVID-19 tienen 6 veces más riesgo de presentar una mala calidad de vida en el trabajo (OR: 6,76; IC 95 \%: 1,51-30,12). De igual manera, las personas que no pueden dormir por estar preocupados por la COVID-19 tuvieron 5 veces más riesgo de presentar una mala calidad de vida en el trabajo (OR: 5,51; IC 95 \%: 1,10-27,49) (Tabla 4). 


\section{Tabla 1}

Características sociolaborales de la población de estudio

\begin{tabular}{|c|c|c|c|}
\hline Variables & & $n=245$ & $\%$ \\
\hline \multirow[t]{2}{*}{ Sexo } & Femenino & 167 & 68,2 \\
\hline & Masculino & 78 & 31,8 \\
\hline Edad (años) & $\mathrm{Me} \pm \mathrm{DS}$ & \multicolumn{2}{|c|}{$38,6 \pm 8,5$} \\
\hline \multirow[t]{2}{*}{ Estado civil } & Casado/Conviviente & 152 & 62,0 \\
\hline & Soltero/Divorciado & 93 & 38,0 \\
\hline \multirow[t]{3}{*}{ Profesión } & Médico & 64 & 26,1 \\
\hline & Licenciado(a) & 112 & 45,7 \\
\hline & Técnico & 69 & 28,2 \\
\hline \multirow[t]{2}{*}{ Condición laboral } & CAS/Terceros & 145 & 59,2 \\
\hline & Contratado/Nombrado & 100 & 40,8 \\
\hline $\begin{array}{l}\text { Tiempo laborando en la institución } \\
\text { (años) }\end{array}$ & $\mathrm{Me} \pm \mathrm{DS}$ & & \\
\hline \multirow[t]{2}{*}{ Área de trabajo } & Unidades no críticas & 153 & 62,4 \\
\hline & Unidades críticas & 92 & 37,6 \\
\hline \multirow[t]{2}{*}{ Segundo trabajo } & Sí & 86 & 35,1 \\
\hline & No & 159 & 64,9 \\
\hline
\end{tabular}


Tabla 2

Análisis descriptivo de las variables: medidas de bioseguridad, miedo a la COVID-19 y calidad de vida en el trabajo en profesionales de salud del Hospital Huaycán, 2021

\begin{tabular}{|c|c|c|c|}
\hline Variables & & $n=245$ & $\%$ \\
\hline Medidas de bioseguridad & $\begin{array}{l}\text { No cumple } \\
\text { Sí cumple }\end{array}$ & $\begin{array}{c}94 \\
151\end{array}$ & $\begin{array}{l}38,4 \\
61,6\end{array}$ \\
\hline \multirow[t]{2}{*}{ Calidad de vida en el trabajo } & Mala & 200 & 81,6 \\
\hline & Buena & 45 & 18,4 \\
\hline \multirow[t]{2}{*}{ Condiciones de trabajo } & Mala & 141 & 57,6 \\
\hline & Buena & 104 & 42,4 \\
\hline \multirow[t]{2}{*}{ Exigencias trabajo-vida } & Mala & 204 & 83,3 \\
\hline & Buena & 41 & 16,7 \\
\hline \multirow[t]{2}{*}{ Clima hacia la seguridad } & Mala & 175 & 71,4 \\
\hline & Buena & 70 & 28,6 \\
\hline \multirow[t]{2}{*}{ Discriminación } & Mala & 231 & 94,3 \\
\hline & Buena & 14 & 5,7 \\
\hline \multirow[t]{2}{*}{ Ergonomía } & Mala & 216 & 88,2 \\
\hline & Buena & 29 & 11,8 \\
\hline \multirow[t]{2}{*}{ Salarios y beneficios } & Mala & 222 & 90,6 \\
\hline & Buena & 23 & 9,4 \\
\hline \multirow[t]{2}{*}{ Hostigamiento } & Mala & 237 & 96,7 \\
\hline & Buena & 8 & 3,3 \\
\hline \multirow[t]{2}{*}{ Flexibilidad } & Mala & 212 & 86,5 \\
\hline & Buena & 33 & 13,5 \\
\hline \multicolumn{4}{|l|}{ Miedo al COVID-19 } \\
\hline \multirow[t]{2}{*}{ Tengo mucho miedo de la COVID-19 } & No & 138 & 56,3 \\
\hline & Sí & 107 & 43,7 \\
\hline \multirow{2}{*}{ Me pone incómodo(a) pensar en la COVID-19 } & No & 215 & 87,8 \\
\hline & Sí & 30 & 12,2 \\
\hline Mis manos se ponen húmedascuando pienso & No & 232 & 94,7 \\
\hline en la COVID-19 & Sí & 13 & 5,3 \\
\hline Tengo miedo de perder mi vida acausa de la & No & 127 & 51,8 \\
\hline COVID-19 & Sí & 118 & 48,2 \\
\hline Cuando veo noticias e historias sobre la COVID- & No & 227 & 92,7 \\
\hline $\begin{array}{l}19 \text { en redes sociales me pongo nervioso(a) o } \\
\text { ansioso(a) }\end{array}$ & Sí & 18 & 7,3 \\
\hline No puedo dormir porque estoy preocupado de & No & 232 & 94,7 \\
\hline contagiarme de COVID-19 & Sí & 13 & 5,3 \\
\hline Mi corazón se acelera o palpita cuando pienso & No & 232 & 94,7 \\
\hline en contagiarme de COVID-19 & Sí & 13 & 5,3 \\
\hline
\end{tabular}


Tabla 3

Análisis bivariado de las características generales, medidas de bioseguridad y miedo al COVID-19

según la calidad de vida en el trabajo en profesionales de salud del Hospital Huaycán, 2021

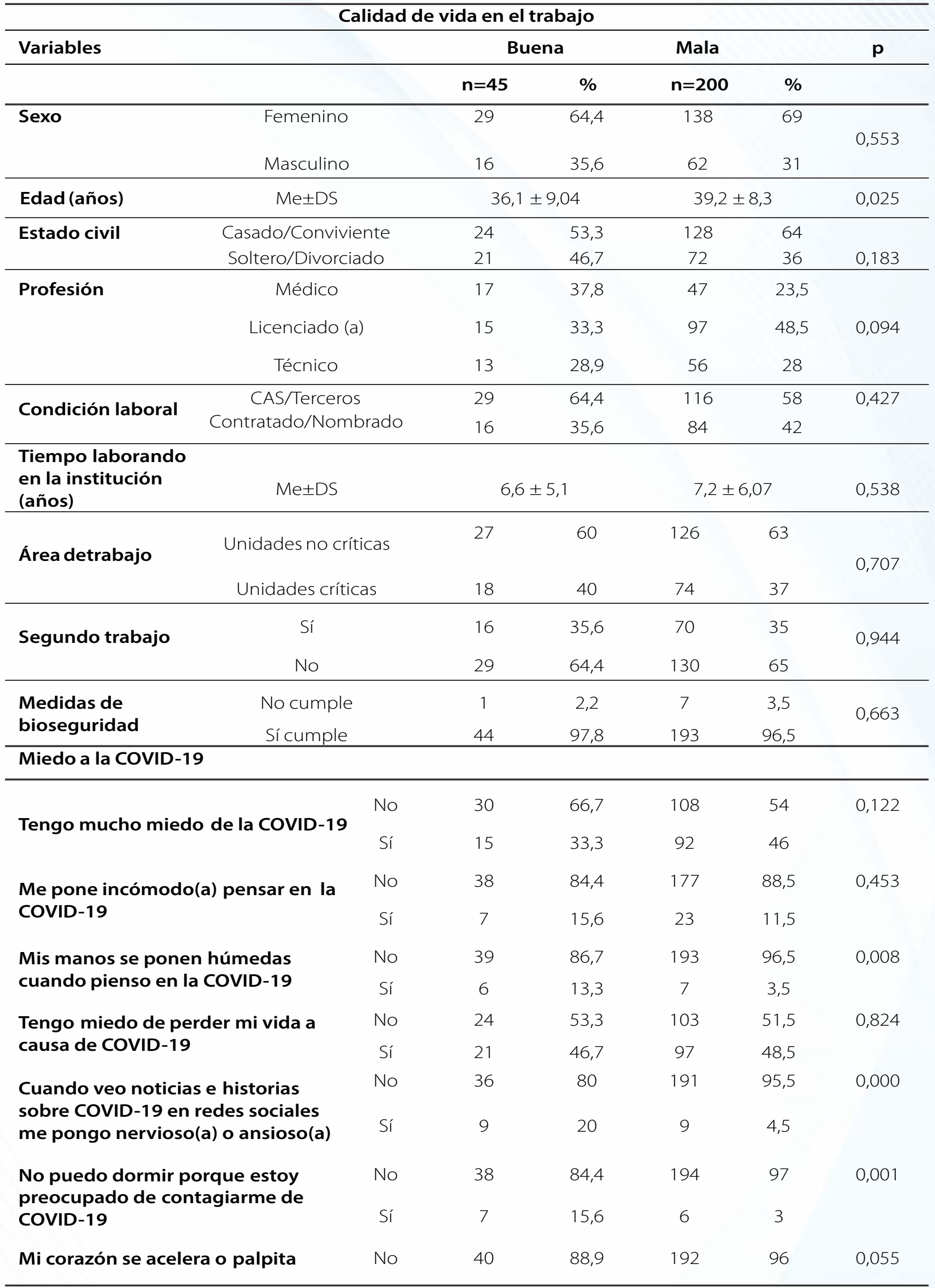


Análisis multivariado de las variables: características generales, medidas de bioseguridad, miedo a la COVID-19 según la calidad de vida en el trabajo en profesionales de salud del Hospital Huaycán, 2021

\begin{tabular}{|c|c|c|c|c|}
\hline \multirow{2}{*}{ Variables } & \multirow[t]{2}{*}{ OR } & \multicolumn{2}{|c|}{ IC $95 \%$} & \multirow[t]{2}{*}{ Sig. } \\
\hline & & Inferior & Superior & \\
\hline Sexo & 0,883 & 0,376 & 2,078 & 0,776 \\
\hline Edad & 0,969 & 0,915 & 1,026 & 0,281 \\
\hline Estado civil & 1,081 & 0,505 & 2,317 & 0,840 \\
\hline \multicolumn{5}{|l|}{ Profesión } \\
\hline Médico & 1,401 & 0,531 & 3,696 & 0,496 \\
\hline Licenciado(a) & 0,675 & 0,278 & 1,635 & 0,383 \\
\hline Condición laboral & 1,279 & 0,502 & 3,259 & 0,606 \\
\hline Tiempo laborando en la institución(años) & 0,988 & 0,904 & 1,079 & 0,784 \\
\hline Área de trabajo & 1,044 & 0,475 & 2,297 & 0,914 \\
\hline Segundo trabajo & 1,132 & 0,504 & 2,544 & 0,764 \\
\hline Medidas de bioseguridad & 2,437 & 0,228 & 26,056 & 0,461 \\
\hline Tengo mucho miedo del COVID-19 & 0,440 & 0,173 & 1,121 & 0,085 \\
\hline Me pone incómodo(a) pensar enCOVID-19 & 0,326 & 0,066 & 1,621 & 0,171 \\
\hline $\begin{array}{l}\text { Mis manos se ponen húmedas cuando pienso en el } \\
\text { COVID-19 }\end{array}$ & 3,070 & 0,447 & 21,065 & 0,254 \\
\hline $\begin{array}{l}\text { Tengo miedo de perder mi vida a causa del COVID- } \\
19\end{array}$ & 1,489 & 0,617 & 3,589 & 0,376 \\
\hline Cuando veo noticias e historias sobre el COVID-19 & & & & \\
\hline $\begin{array}{l}\text { en redes sociales me pongo nervioso (a) o } \\
\text { ansioso(a) }\end{array}$ & 6,761 & 1,518 & 30,120 & 0,012 \\
\hline $\begin{array}{l}\text { No puedo dormir porque estoy preocupado de } \\
\text { contagiarme deICOVID-19 }\end{array}$ & 5,510 & 1,104 & 27,496 & 0,037 \\
\hline $\begin{array}{l}\text { Mi corazón se acelera o palpita cuando pienso en } \\
\text { contagiarme deICOVID-19 }\end{array}$ & 0,470 & 0,055 & 4,011 & 0,490 \\
\hline
\end{tabular}

\section{Discusión}

Los profesionales de la salud tienden a experimentar una peor calidad de vida debido a las pesadas cargas de trabajo, la rotación por turnos, el sufrimiento de los pacientes y muchos otros factores ocupacionales tienen un papel crucial en la protección de la salud en los nosocomios. Sumado a ello, las medidas de restricción y aislamiento dadas por el estado para el control de la infección por COVID-19 ha destapado la pésima calidad de vida en el trabajo que presentan los profesionales de la salud. ${ }^{22}$

La investigación encontró que el 81,6 \% de los profesionales de la salud calificó su calidad de vida en el trabajo como mala y solo el 18,4\% como buena. Resultados similares obtuvo un 
estudio durante el brote de Ébola en África que informó una disminución significativa en la salud física y la calidad de vida psicológica de los trabajadores de salud, ${ }^{23}$ de igual manera un estudio en Países Bajos indicó que la calidad de vida autoinformada de los trabajadores de la salud fue significativamente menor durante el pico de COVID-19, en comparación a antes del inicio de la pandemia. ${ }^{24}$ En comparación con el público, los trabajadores de la salud son altamente susceptibles a respuestas psicológicas negativas exacerbadas por el riesgo de contacto con pacientes. Además de ello, la falta de una práctica basada en la evidencia relacionada con el manejo de pacientes con COVID-19 y la desinformación sobre el tema merman la calidad de vida de los trabajadores. ${ }^{25}$

Otro resultado importante que se encontró en esta investigación, fue que el $61,6 \%$ de los profesionales de salud sí cumple con las medidas de bioseguridad para la prevención del COVID-19 dentro del hospital; mientras que el $38,4 \%$ no las cumple (Tabla 2). Resultados similares se encontraron en un estudio hecho en China, ${ }^{26}$ en el cual, el 89,7\% de los trabajadores de salud siguió las prácticas correctas de prevención de COVID-19. A contraposición, en Brasil, ${ }^{27}$ el 95,9 \% de médicos odontólogos informó tener conocimiento de las normativas de prevención de COVID-19 dadas por el estado, sin embargo, en cuanto a prácticas, su nivel de acatamiento de las medidas de bioseguridad fue sólo del $28 \%$. La literatura señala que la COVID19 es una enfermedad muy transmisible; sus mecanismos son principalmente gotitas respiratorias, partículas aerosolizadas y por contacto. Además de ello, algunos procedimientos están asociados con la producción de aerosoles y la mayoría de los pacientes presentan síntomas leves y un 10-30\% necesita hospitalización en la $\mathrm{UCl}^{27}$ Entonces es innegable, que los profesionales de la salud tienen una alta probabilidad de contagio por COVID-19, lo que hace necesario que los sistemas médicos garanticen la reducción del riesgo de infección entre el personal de la salud que están o no en contacto directo con los pacientes, a través de la implementación de políticas y educación para transmitir la importancia de las medidas de bioseguridad. ${ }^{28}$
En lo que respecta al miedo por COVID-19, se encontró que los profesionales de salud que tienen miedo cuando ven noticias o historias sobre la COVID-19 tienen 6 veces más riesgo de presentar una mala calidad de vida en el trabajo (OR: 6,761; IC $95 \%=1,51-30,12$ ). De igual manera, las personas que no pueden dormir por estar preocupados por la COVID-19 tuvieron 5 veces más riesgo de presentar una mala calidad de vida en el trabajo (OR: 5,510; IC $95 \%=1,10-$ 27,49) (Tabla 4). Al igual, un estudio en Turquía ${ }^{22}$ hecho en enfermeras hospitalarias, reveló que el miedo a la COVID-19 afecta de forma negativa la calidad de vida laboral. Del mismo modo, un estudio en Egipto ${ }^{29}$ reveló que el miedo percibido a la infección por COVID-19 se correlaciona negativamente con los logros personales y todo lo que embarga la calidad de vida. Además, un estudio en Jordania ${ }^{30}$ reveló que los trabajadores de salud tuvieron altos niveles de miedo a la COVID-19 y esto se asoció positivamente a la ansiedad, depresión y estrés.

La exposición prolongada al estrés, miedo o ansiedad da como resultado un agotamiento emocional y se manifiesta como falta de entusiasmo en el trabajo, impotencia, sensación de estar atrapado y abrumado. Los profesionales de la salud pueden desarrollar una actitud negativa hacia sus colegas, tratar con indiferencia a sus pacientes o ser apartados de sus responsabilidades profesionales, lo que los lleva a un estado de despersonalización y falta de realización personal. ${ }^{31}$

Numerosos estudios confirmaron que la calidad de vida en el trabajo se ha visto afectada durante la pandemia por la COVID-19. Esto debido a la exposición a altos niveles de angustia y miedo en el trabajo, el agotamiento emocional, angustia mental y/o física. Las consecuencias negativas de este fenómeno generan una mayor incidencia de errores médicos e insatisfacción laboral, e incluso una jubilación anticipada. Finalmente, la revisión de la literatura señala que los trabajadores de salud que no pueden tolerar las cargas de trabajo son más propensos a informar una mala calidad de vida, particularmente en los dominios físicos y psicológicos de sus vidas. ${ }^{32}$ 


\section{Referencias}

1. Organización Mundial de la Salud [OMS]. WHO Coronavirus Disease (COVID-19) Dashboard. 23 dejulio. 2020.

2. Organización Panamericana de la Salud O. Brote de enfermedad por el Coronavirus (COVID-19). 28 de julio. 2020.

3. Ministerio de Salud. Casos confirmados por coronavirus COVID-19 ascienden a 371,096 en el Perú. 23 de julio. 2020.

4. OPS/OMS. Cerca de 570.000 trabajadores de la salud se han infectado y 2.500 han muerto por COVID-19 en las Américas - OPS/OMS | Organización Panamericana de la Salud. 2020

5. Devica S. Centro Nacional de Epidemiología, Prevención y Control de Enfermedad [Internet]. Vol. 7, Ministerio de Salud. 2020 . Available from: https://www.dge.gob.pe/nindex.php

6. Lippi G, Adeli K, Adeli K, Ferrari M, Horvath $A R$, Koch D, et al. Biosafety measures for preventing infection from COVID-19 in clinical laboratories: IFCC Taskforce Recommendations. Vol. 58, Clinical Chemistry and Laboratory Medicine. De Gruyter;2020.p. 1053-62.

7. WuY,Wang J, Luo C, Hu S, Lin X, Anderson AE, et al. A Comparison of Burnout Frequency Among Oncology Physicians and Nurses Working on the Frontline and Usual Wards During the COVID-19 Epidemic in Wuhan, China. J Pain Symptom Manage. 2020 Jul;60(1):e60-5.

8. Lai J, Ma S, Wang Y, Cai Z, Hu J, Wei N, et al. Factors associated with mental health outcomes among health care workers exposed to Coronavirus Disease 2019. JAMA Netwopen. 2020 Mar;3(3): e203976.

9. Foro Internacional de Medicina Interna. Ataque al personal de la salud durante la pandemia de COVID-19 en Latinoamérica. Educy Práctica la Med.2020;45(3).

10. Brooks SK, Webster RK, Smith LE, Woodland L, Wessely S, Greenberg N, et al. The psychological impact of quarantine and how to reduce it: rapid review of the evidence. Vol. 395, The Lancet. Lancet Publishing Group;2020. p. 912-20.

11. Brooks S, Amlôt R, Rubin GJ, Greenberg N. Psychological resilience and post-traumatic growth in disaster-exposed organisations: Overview of the literature. J R Army Med Corps. 2018 Feb;166(1).

12. Xiang YT, Yang Y, Li W, Zhang L, Zhang Q, Cheung T, et al. Timely mental health care for the 2019 novel coronavirus outbreak is urgently needed. The Lancet Psychiatry. 2020; 7:228-9.

13. Shigemura J, Ursano RJ, Morganstein JC, Kurosawa M, Benedek DM. Public responses to the novel 2019 coronavirus (2019-nCoV) in Japan: Mental health consequences and target populations. Psychiatry and Clinical Neurosciences. Blackwell Publishing; 2020; 74:281-2.

14. Reardon S. Ebola's mental-health wounds linger in Africa: Health-care workers struggle to help people who have been traumatized by the epidemic. Nature. Nature Publishing Group; 2015. Vol. 519, p. 13-4.

15. Seçer I, Ulaş S, Karaman-Özlü Z. The Effect of the Fear of COVID-19 on Healthcare Professionals' Psychological Adjustment Skills: Mediating Role of Experiential Avoidance and Psychological Resilience. Front Psychol. 2020 Oct;11.

16. Shreffler J, Petrey J, Huecker M. The impact of COVID-19 on healthcare worker wellness: A scoping review. West J Emerg Med. 2020;21(5):1059-66.

17. Ministerio de Salud. Plan nacional de reforzamiento de los servicios de salud y contención del COVID-19. Documento técnico. Resolución Ministerial № 95-2020MINSA. 2020. Lima, Perú.

18. Ministerio del interior. Plan para la vigilancia, prevención y control de la COVID-19 en el trabajo. Versión 3. Proyecto Especial CORAH. Actualizado de acuerdo a la Resolución Ministerial Nº 972-2020-MINSA. 2021. Lima, Perú.

19. Ahorsu DK, Lin CY, Imani V, Saffari M, Griffiths MD, Pakpour AH. The Fear of COVID-19 Scale: Development and Initial Validation. Int J Ment Health Addict. 2020 Mar 27:1-9. doi: 10.1007/s11469-020-00270-8.

20. Huarcaya-Victoria J, Villarreal-Zegarra D, Podestà A. et al. Psychometric Properties of a Spanish Version of the Fear of COVID-19 Scale in General Population of Lima, Peru. Int J Ment Health Addiction. 2020; 22: 1-14. 
https://doi.org/10.1007/s11469-02000354-5

21. Moreno I, Cuevas M, González J.Validación y adaptación del cuestionario de calidad de vida en el trabajo (QWLQ) y del cuestionario genérico de estrés en el trabajo (GJSQ). Revista Interamericana de Psicología Ocupacional. 2009; 28(1):56-80.

22. Maslakçı A, Sürücü L, Sesen H. Fear ofCOVID19 and work-quality of life among nurses: The mediating role of psychological wellbeing. Manag Sci Lett. 2021; 11:1985-90.

23. Jones S, White S, Ormrod J, Sam B, Bull F, Pieh $S$, et al. Work-based risk factors and quality of life in health care workers providing maternal and newborn care during the Sierra Leone Ebola epidemic: Findings using the WHOQOL-BREF and HSE Management Standards Tool. BMJ Open [Internet]. 2020 Nov 14 [cited 2021 May 25];10(11):32929. Available from:http://bmjopen.bmj.com/

24. Kheiraoui F, Gualano MR, Mannocci A, Boccia A, La Torre G. Quality of life among healthcare workers: A multicentre crosssectional study in Italy. Public Health. 2012; 126(7):624-9.

25. Boluarte A, Sánchez A, Rodríguez A, Merino C. Working conditions and emotional impact in healthcare workers during COVID19 pandemic [Internet]. Journal of Healthcare Quality Research. 2020; 35: $401-2$. A va ila ble from: https://www.elsevier.es/en-revista-journalhealthcare-quality- research-257-articuloworking-conditions-emotional-impact-inS2603647920300919

26. Zhang $M$, Zhou $M$, Tang $F$, Wang $Y$, Nie $H$, Zhang $L$, et al. Knowledge, attitude, and practice regarding COVID-19 among healthcare workers in Henan, China. J Hosp Infect [Internet]. 2020;105(2):183-7. A $\vee$ a i $|\mathrm{a} b| \mathrm{e}$ from: /pmc/articles/PMC7194961/

27. Morais HG de F, Galvão MHR, Silva WR da, Barros JM de, Santos AA dos, Domingos NR da S, et al. Biosafety knowledge, actions and practices of brazilian dentists during the COVID-19 pandemic. Res Soc Dev. 2020;9(10): e1529108507.

28. Díaz-Guio DA, Díaz-Guio Y, Pinzón-Rodas V, Díaz-Gomez AS, Guarín- Medina JA,
Chaparro-Zúñiga Y, et al. COVID-19: Biosafety in the Intensive Care Unit [Internet]. Current Tropical Medicine Reports. Springer Science and Business Media Deutschland GmbH; 2020; 7:104-11. A v a i l a ble from : https://doi.org/10.1007/s40475-02000208-z

29. Abdelghani M, El-Gohary HM, Fouad E, Hassan MS. Addressing tthe relationship between perceived fear of COVID-19 virus infection and emergence of burnout symptoms in a sample of Egyptian physicians during COVID-19 pandemic: a cross-sectional study. Middle East Curr Psychiatry [Internet]. 2020;27(1):1-9. A $\vee$ a i l a ble from : https://doi.org/10.1186/s43045-02000079-0

30. Alnazly E, Khraisat OM, Al-Bashaireh AM, Bryant CL. Anxiety, depression, stress, fear and social support during COVID-19 pandemic among Jordanian healthcare workers. PLoS One [Internet]. 2021;16: e 0247679 . Available from: https://doi.org/10.1371/journal.pone.0247 679

31. Al Dhaheri AS, Bataineh MF, Mohamad MN, Ajab A, Al Marzouqi A, Jarrar AH, et al. Impact of COVID-19 on mental health and quality of life: ¿ls there any effect? A crosssectional study of the MENA region. PLoS One [Internet]. 2021;16: e0249107. Available f $r \quad 0 \quad m$

https://doi.org/10.1371/journal.pone.0249 107

32. Young KP, Kolcz DL, O'Sullivan DM, Ferrand J, Fried J, Robinson K. Health care workers' mental health and quality of life during COVID-19: Results from a midpandemic, national survey. Psychiatr Serv [Internet]. 2021;72(2):122-8. A va il a b l e from: https://ps.psychiatryonline.org/doi/abs/10. 1176/appi.ps.202000424.

\section{Correspondencia:}

obedlopez.21.ol@gmail.com

Fecha de recepción: 13/8/2021

Fecha deaceptación:26/11/2021 